\section{Promoción de habilidades socioemocionales para la resolución de conflictos y conductas de riesgo en adolescentes en condiciones de vulnerabilidad social}

\author{
Promotion of socio-emotional skills for the resolution of conflicts and risk behaviors in \\ adolescents in conditions of social vulnerability
}

Marisol Morales Rodríguez

\section{RESUMEN}

Las condiciones de vulnerabilidad social afectan el sano desarrollo de los individuos de diversas comunidades, pero en particular los adolescentes, constituyen una población de riesgo dados los cambios a los que se enfrentan, los cuales se agudizan en contextos adversos; por ello la necesidad de implementar acciones tendientes a disminuir conductas de riesgo que permitan enfrentarse eficazmente a los desafíos diarios y promover comportamientos adaptativos. El objetivo del estudio fue promover mediante una intervención, habilidades socioemocionales asociadas a la resolución de conflictos en adolescentes en condiciones de vulnerabilidad, para disminuir conductas riesgo, en particular la actitud ante drogas y alcohol. Basado en una metodología cuantitativa, se utilizó un diseño preexperimental; participaron 69 adolescentes escolarizados en un programa de intervención con duración de 3 meses; se utilizaron las escalas Tolerancia a la frustración, Autoeficacia, Conflictack, Resistencia a la presión de amigos para consumo de alcohol y cuestionario de actitud ante drogas. Los resultados muestran cambios significativos en Tolerancia a la frustración, Actitud ante drogas y alcohol y Resolución de problemas. Se concluye que, al desarrollar habilidades socioemocionales, los adolescentes aprendieron a enfrentar eficazmente situaciones problemáticas, mostrando una actitud de rechazo hacia el alcohol y las drogas y con ello, reduciéndose la probabilidad de involucrarse en adicciones.

Palabras clave: Habilidades socioemocionales; Resolución de conflictos; Conductas de riesgo; Adolescentes; Programa de intervención

\section{ABSTRACT}

Conditions of social vulnerability affect the healthy development of individuals from various communities, but particularly adolescents, constitute a population at risk given for the changes they face, which are exacerbated in adverse contexts; for this reason, they need to implement actions to reduce risky behaviors that allow to deal effectively with daily problems and promote adaptive behaviors. The objective of the study was to promote through an intervention, socio-emotional skills associated with conflict resolution in adolescents in vulnerable conditions, to reduce risk behaviors, in particular the attitude towards drugs and alcohol. Based on a quantitative methodology, a preexperimental design was used; 69 adolescents enrolled in the school participated in an intervention program lasting 3 months; The scales Tolerance to frustration, Selfefficacy, Conflictack, Resistance to pressure from friends for alcohol consumption, and a questionnaire on attitude towards drugs were used. The results show significant changes in Tolerance to frustration, Attitude towards drugs and alcohol, and Problemsolving. It is concluded that by developing socio-emotional skills, adolescents learned to deal effectively with problematic situations, showing an attitude of rejection towards alcohol and drugs and with it, reducing the probability of getting involved in addictions.

Keywords: Socio-emotional skills; Conflict resolution; Risky behaviors; Adolescents; Intervention program
RELIGACIÓN

REVISTA DE CIENCIAS SOCIALES Y HUMANIDADES JOURNAL OF SOCIAL SCIENCES AND HUMANITIES
REVISTA DE CENCIAS SOCIAIS E HUMANAS

INFORMACIÓN

http://doi.org/10.46652/rgn.v6i30.851 ISSN $2477-9083$

Vol. 6 No. 30, 2021. e210851 Quito, Ecuador

Enviado: octubre 04, 2021

Aceptado: noviembre 28, 2021 Publicado: diciembre 14, 2021 Publicación Continua Sección General | Peer Reviewed

\section{AUTORA:}

(D) Marisol Morales Rodríguez

Universidad Michoacana de San Nicolás de Hidalgo - México marisol.morales@umich.mx

Conflicto de intereses

La autora declara que no existe conflicto de interés posible.

Financiamiento

No existió asistencia financiera de partes externas al presente artículo.

Agradecimiento

A la Telesecundaria 178 de Morelia, Michoacán por permitir la implementación de la intervención y por su disposición a lo largo del proceso.

Nota

El artículo no se desprende de un trabajo anterior.

ENTIDAD EDITORA

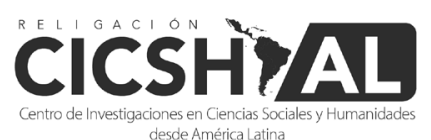




\section{Introducción}

La adolescencia es una etapa caracterizada por una diversidad de cambios en las distintas áreas del desarrollo que preparan al individuo para que en un futuro próximo se inserte en el mundo adulto. Según Valdés y Ochoa (2010) señalan que las profundas alteraciones biológicas, psicológicas y sociales, son generadoras de conflictos y contradicciones, no obstante, es un período de adaptación, también es una fase de grandes determinaciones que llevan al individuo a una mayor independencia psicológica y social.

Las fuertes transformaciones obligan al adolescente a utilizar diversos recursos personales para hacerle frente a los desafíos. Entre estos destacan las habilidades socioemocionales, las cuales según Aranda y Caldera (2018) son capacidades que favorecen el autoconocimiento, el manejo eficaz de las emociones, la comunicación efectiva, la toma de decisiones, resolver conflictos, plantear y alcanzar metas, así como el manejo del estrés. En tanto, Trejo y Hernández (2018) señalan que dichas habilidades son capacidades que engloban el entendimiento y regulación de emociones, el desarrollo de relaciones positivas y la toma de decisiones responsables.

De esta manera, las habilidades socioemocionales pueden ser entendidas como un conjunto de destrezas que favorecen procesos adaptativos, ya que por un lado contribuyen al establecimiento y mantenimiento de relaciones interpersonales satisfactorias, y por otro, a un manejo eficaz de las emociones, incluyendo procesos de autorregulación.

En la misma línea, Bisquerra (2003) asevera que equivalen a un conjunto de conocimientos, capacidades, habilidades y actitudes que permiten la comprensión, expresión y regulación efectiva de fenómenos emocionales propios y de los demás. Complementando lo anterior, Aranda y Caldera (2018) afirman que dichas habilidades son herramientas que permiten conocerse mejor como personas, manejar las emociones propias, comunicarse de manera efectiva, resolver conflictos, plantear y alcanzar metas, manejar el estrés y tomar decisiones reflexivas.

Además de las citadas, intervienen otras características como el autoconocimiento, la conciencia social, colaboración y la perseverancia que definen las habilidades socioemocionales (Treviño et al., 2019). En congruencia con lo anterior, lo que resulta novedoso y prometedor en el estudio de dichas habilidades es que se pueden aprender y mejorar (West, 2016). Y si son susceptibles de desarrollarse, incrementan el bienestar personal a partir de su mejoramiento, utilizando estrategias psicoeducativas con adolescentes.

Diversos estudios muestran las ventajas de implementar programas de intervención tendientes a desarrollar las habilidades socioemocionales en adolescentes como el de Garaigordobil (2008) o el programa Social and Emotional Aspects of Learning for secondary schools (SEAL) en Reino Unido, destacando mejora en el rendimiento académico, mejor resolución de problemas, mayor motivación intrínseca, mejor comportamiento en clase y una cultura de la escuela más positiva (Rodríguez et al., 2012).

En la misma línea, un estudio reportó a través de una intervención con adolescentes, basada en una metodología vivencial y participativa, confirmaron que la intervención basada en una educación emocional, trae consigo una serie de beneficios como el mejoramiento del bienestar 
emocional y social, la capacidad para analizar sentimientos propios y ajenos, y el desarrollo de una gama de habilidades sociales (Mónaco et al., 2017), lo que favorecerá en los adolescentes, enfrentar con mayor éxito sus propios cambios y las exigencias diarias. Aunado a lo anterior, Duckworth y Yeager (2015) reportan que estudios longitudinales han corroborado que las capacidades sociales y emocionales que facilitan el logro de metas, relaciones sociales saludables y la toma de decisiones, predicen el desempeño social, psicológico y el bienestar en general.

Confirmando lo antes dicho, las habilidades sociales y emocionales son susceptibles de desarrollarse, por influencia de diversos factores entre los que destaca la educación (García, 2018), lo que abre la puerta para utilizar los espacios educativos para promoverlas e implementar acciones creativas como los programas de intervención para lograr el objetivo. Rodríguez et al., (2012) señalan que uno de los elementos clave de este tipo de programas de intervención son las dinámicas grupales y los ejercicios cooperativos, lo que favorece un espacio de reflexión, pero también permiten el establecimiento de un clima de clase adecuado y la resolución pacífica de conflictos.

Hablando de resolución pacífica de conflictos, diversas investigaciones han resaltado que constituye un aspecto crucial para favorecer relaciones interpersonales satisfactorias. Es importante partir de la conceptualización del conflicto, el cual es inevitable, inherente a la vida en sociedad. Particularmente, el conflicto forma parte de las interacciones cotidianas del adolescente, lo que le permite a su vez, desarrollar una mayor capacidad de anticipación, autorregulación, negociación y empatía. El interés por estudiar el conflicto radica en identificar la manera en que el adolescente lo enfrenta y resuelve; un conflicto será resuelto eficazmente, en la medida en que el resultado sea adaptativo.

Los conflictos según Rojas-Solís et al., (2019) se conciben como la principal causa de ruptura en cualquier tipo de relación interpersonal, lo que genera un sentimiento de malestar; de ahí la trascendencia de emplear estrategias positivas de resolución, lo que tiende a la reducción de conductas violentas y malestar emocional, y, por lo tanto, el aumento del bienestar personal.

Garaigordobil et al., (2016) afirman que la percepción que se tiene del conflicto constituye la base en función de lo cual será afrontado. Los adolescentes con una actitud positiva hacia el conflicto, esto es, basada en la negociación y orientada hacia el problema, serán más capaces de afrontar y resolver los conflictos de manera adecuada, a diferencia de aquellos que tengan una actitud negativa, evitándolo o respondiendo de manera agresiva, ya que finalmente no resolverán.

La resolución de conflictos involucra un proceso cognitivo-conductual que se lleva a cabo internamente para afrontar de manera adaptativa situaciones que demandan respuestas eficaces (Merino, 2012). Implica un proceso de toma de decisiones, donde se ponderan las alternativas y se elige. La elección lleva a la acción y es cuando se observa el estilo con el que se resuelven los problemas. Berdullas (2016) plantea que a medida que la capacidad de solucionar los conflictos es mayor, aumenta el bienestar emocional del adolescente, lo que, a su vez favorece patrones de comportamiento más adaptativos.

Así, se aprecia que detrás de dicho proceso resolutivo se entreteje una serie de estrategias que incluyen la planificación, el análisis, la anticipación, la evaluación y diversas funciones 
superiores que favorecen la puesta en marcha de acciones tendientes a obtener el éxito. De esta manera, la capacidad de resolver conflictos se relaciona significativamente con las habilidades socioemocionales, las cuales, en palabras de Marrero et al., (2018) favorecen la comunicación adecuada, el autocontrol y la autoconfianza. Dichas premisas llevan a una reflexión importante, que Treviño et al., (2019) sacan a la luz, al afirmar que las decisiones que toman las personas, las cuales muchas de las veces se asocian a la solución de un problema, determinan la forma de vivir.

La forma de vida de una persona está condicionada además de factores personales, por condicionantes externos. Existen contextos sociales poco propicios para el desarrollo de sus habitantes, en particular los adolescentes, colocándolos en una situación desfavorecida o de vulnerabilidad. Este término aplica a estar en desventaja y, hablar de vulnerabilidad social plantea la existencia de diversas condiciones ambientales y sociales ponen al adolescente y en general a todos los individuos de una comunidad, en condiciones de riesgo. Cabe aclarar que si bien la pobreza es un de los más potentes indicadores de vulnerabilidad social no es sinónimo de esta, ya que además intervienen otros condicionantes económicos, sociales y culturales.

Desde esta lógica, Ortiz-Ruiz y Díaz-Grajales (2018) afirman que la vulnerabilidad social se define como las condiciones de desventaja y rezago social, económico y cultural que experimentan diversos grupos sociales. Las comunidades que se caracterizan por inseguridad, incertidumbre y desprotección y cuya fuente puede ser de cualquier ámbito llámese económico, social, religioso o ético, se convierten en un riesgo evidente para quienes las habitan, de tal manera que actúan como un potente factor de riesgo (Sánchez-González \& Egea-Jiménez, 2012).

Los factores de riesgo según Moreta et al., (2017) son aquellos elementos que surgen de cualquier tipo de circunstancia de naturaleza biológica, psicológica, social o medioambiental, cuya presencia o ausencia influye en la posibilidad de que se presente un problema en el desarrollo de un individuo o un grupo social. Ha destacado que dichos factores están compuestos por dos elementos: la amenaza y la vulnerabilidad. La primera corresponde a un fenómeno, sustancia, actividad humana o condición que puede ocasionar un impacto negativo en la salud; y la vulnerabilidad tiene que ver con la "capacidad disminuida de una persona para anticiparse, confrontar y tolerar los efectos de un daño para su posterior recuperación” (p. 32). Como tal, los factores de riesgo a su vez pueden llevar a la aparición de conductas de riesgo, las cuales colocan al adolescente en una condición adversa ya que atentan contra su calidad de vida y bienestar personal.

Las conductas de riesgo son aquellas acciones voluntarias o involuntarias que lleva a cabo una persona o grupo de personas y que tienen consecuencias nocivas. En su desarrollo contribuyen rasgos que se asocian a la edad propiamente como en el caso de la adolescencia, en particular la sensación de invulnerabilidad, la búsqueda de sensaciones novedosas, la presión de los iguales, la necesidad de autonomía creciente y reafirmación de la identidad (Rosabal et al., 2015). Tales conductas se ven reflejadas en embarazos a temprana edad, delincuencia, adicciones, trastornos en la salud mental, entre otros.

Tales conductas suelen ser reforzadas por la vulnerabilidad social, como ya fue señalado anteriormente, ya que en buena medida se relaciona con las posibilidades de acceso que tienen 
los adolescentes a la drogas lícitas e ilícitas, lo cual constituye una seria amenaza para su desarrollo. La vulnerabilidad asociada a la exposición y consumo de sustancias psicoactivas suele ser muy común en comunidades marginadas donde además de la pobreza, hay limitados servicios y opciones de esparcimiento para los jóvenes, inseguridad, violencia, aunado a la ausencia de límites y reglas tanto familiares como sociales.

Tal es el caso del contexto en el cual se llevó a cabo el estudio, el cual se caracteriza por la existencia de condiciones mencionadas anteriormente, según lo reportado por docentes de la institución educativa donde se llevó a cabo la investigación; dicha información fue corroborada por los mismos adolescentes y padres de familia, así como por la observación realizada por parte del investigador. La comunidad se encuentra ubicada en la periferia de la Ciudad de Morelia, Michoacán, México y según sus habitantes aseguran que existe un aumento de la violencia y la delincuencia en los últimos 10 años, existe mayor inseguridad y una exposición repetida al consumo de sustancias psicoactivas, lo que genera un círculo vicioso. Al haber pocos espacios de esparcimiento, aunado de una escasa supervisión parental, los jóvenes se involucran en conductas de riesgo, perpetuándose los altos índices de delincuencia e inseguridad.

Lo anterior ha sido constatado por diversas fuentes; de acuerdo con los datos de la Encuesta Nacional de Seguridad Pública Urbana de 2018 publicada en 90 grados (2019), el 70\% de la población mayor de 18 años en el municipio de Morelia se siente insegura. Tal percepción suele ser compartida por los habitantes de la comunidad, la cual está inserta en el municipio de Morelia, al asegurar que incluso hay venta de drogas, hallazgos de muertos e intentos de secuestro. Pero de manera particular, un fenómeno que sobresale es la exposición abierta de consumo de alcohol y drogas ilegales, lo que atrae el interés de los adolescentes, por lo que se teme que se involucren en conductas de alto riesgo.

Existen diversos postulados teóricos que abordan la actitud de disposición al consumo de drogas legales e ilegales, señalando que la motivación hedónica, la presión social de los iguales y de la comunidad, así como la búsqueda de sensaciones, son algunos de los factores que contribuyen a preservar dicha actitud en los adolescentes (Alarcón et al., 2015). Las actitudes predisponen a determinados comportamientos, por lo que, en palabras de Moreta, et al., (2018) generan una reacción valorativa de agrado o de desagrado, que aplicado al consumo de drogas pueden convertirse en un factor de riesgo o de protección. A ello se suma, el fenómeno de aprobación social, cobrando sentido el consumo como elemento favorecedor de adaptación social, de pertenencia de grupo, incluso de presión social, lo cual es alimentado también por falta de límites y reglas claras dentro del núcleo familiar.

La oportunidad de consumo se relaciona con una supervisión ineficaz hacia los adolescentes, lo que implica que los agentes de socialización no están llevando a cabo sus funciones principales; y si hay deficiencias en la supervisión existe mayor posibilidad de progresar hacia el consumo de sustancias. Diversas investigaciones han encontrado la existencia de una relación causal con la progresión entre la primera oportunidad de consumo y la primera ocasión en que busca el consumo, de esta manera, la progresión hacia el consumo se relaciona con la posibilidad de acceder fácilmente a ellas (Ruiz \& Medina-Mora, 2014). 
La actitud hacia el consumo de alcohol y drogas ha sido catalogada como una predisposición basada en la percepción de riesgo, relacionada directamente con la intención de realizar la conducta, junto con creencias que surgen en torno a ello. Actualmente, los adolescentes tienen percepciones propias, diferentes a las de los adultos, por lo que es necesario conocer el sentido que le atribuyen al uso de drogas. Desafortunadamente, la baja percepción de riesgo genera un mayor peligro para el consumo de drogas, ya que los adolescentes creen que las drogas no son tan peligrosas y que son capaces de dejarlas en cualquier momento. Si se percibe una droga como riesgo y se valora su consumo como peligroso, la probabilidad de convertirse en consumidor es menor (Melo \& Castanheira, 2010), de ahí la importancia de conocer el significado que poseen de su consumo a fin de contar con bases sólidas para realizar intervenciones.

Los adolescentes que habitan en zonas de alta vulnerabilidad social están expuestos al consumo de sustancias psicoactivas, aunque no necesariamente lleva a su consumo, sin embargo, tales condiciones sociales y la dificultad de lidiar con ello, pueden ser determinantes para que se involucre en conductas de riesgo, de ahí la importancia de que se desarrolle la capacidad de afrontar condiciones adversas y resolver eficazmente los problemas de la vida diaria.

Una vía es mediante la implementación de intervenciones para el desarrollo de habilidades socioemocionales, ya que promueven el afrontamiento eficaz de situaciones imprevistas y adversas, que lleva a la resolución de conflictos, además favorecen actitudes que potencian la prevención de conductas de riesgo como el consumo de drogas y alcohol, al identificar las consecuencias negativas de su uso y abuso, de ahí la trascendencia de dirigir la mirada hacia el constructo en cuestión.

Hernández et al., (2016) afirman que la resolución de problemas constituye una estrategia viable para lograr tal propósito, mediante programas de prevención de drogas. Los programas de intervención han resultado ser táctica efectiva para promover cambios de comportamiento en adolescentes. A partir de lo anterior, surge el siguiente cuestionamiento ¿el fomento de habilidades socioemocionales asociadas al manejo de conflictos disminuye la probabilidad de desarrollar una actitud de aceptación hacia el alcohol y las drogas en adolescentes que viven en condiciones de vulnerabilidad social? Como resultado de la pregunta de investigación, el objetivo general del presente estudio se centró en promover habilidades socioemocionales asociadas a la resolución de conflictos en adolescentes en condiciones de vulnerabilidad social, para disminuir conductas de riesgo, en particular la actitud de aceptación ante las drogas y el alcohol, mediante la implementación de un programa de intervención. Como objetivos particulares se plantearon identificar la relación entre actitud hacia drogas y alcohol y resolución de conflictos; así como la asociación entre la resolución de conflictos y habilidades socioemocionales.

\section{Metodología}

\subsection{Tipo de estudio}

La presente investigación se enmarca en el enfoque cuantitativo. Según Hernández-Sampieri et al., (2010) el enfoque cuantitativo es secuencial y probatorio, esto es, cada fase precede a la siguiente el orden es riguroso. Usa la recolección de datos para probar la hipótesis, se basa en la medición numérica a fin de probar teorías. 


\subsection{Alcance de la investigación}

El alcance es descriptivo-correlacional, ya que, por un lado, se especifican las características del fenómeno de estudio, para posteriormente conocer el grado de asociación entre las variables intervinientes (Hernández-Sampieri, et al., 2010).

\subsection{Diseño del estudio}

El diseño utilizado fue preexperimental, ya que no fue posible contar con grupo control debido a que fueron incluidos en la intervención, los tres grupos de nuevo ingreso por indicaciones de la autoridad educativa; eso mismo evitó que los participantes fueran elegidos al azar.

\subsection{Participantes}

El tipo de muestreo utilizado fue no aleatorio intencional como ya fue puntualizado, ya que la autoridad solicitó que los grupos de nuevo ingreso fueran quienes se beneficiaran del programa de intervención. Cabe destacar que los grupos de segundo y tercer grado ya habían participado en otras intervenciones, por lo tanto, no fueron incluidos. Participaron 69 adolescentes de primer grado, de los cuales el $53 \%$ son mujeres y el $47 \%$ hombres; con edades comprendidas entre los 11 y 13 años ( $M=11.9$ años).

Criterios de inclusión: Adolescentes estudiantes de la Escuela Secundaria 178, con Clave 16ETV0178I, de ambos sexos que cursan el 1er grado, que aceptaron participar voluntariamente y que existe el consentimiento informado por parte de los padres.

Criterios de exclusión: Que los participantes no quisieran participar voluntariamente y la falta de consentimiento informado por parte de los padres. Estudiantes de $2^{\circ}$ y $3 \mathrm{er}$ grado de la misma institución aún cuando no hayan participado en algún programa de intervención previo; así también se excluyó a aquellos que no completaron la evaluación inicial y la post evaluación.

\subsection{Contexto}

El estudio se llevó a cabo en una escuela secundaria inserta en una comunidad que se encuentra en la periferia de la ciudad de Morelia, Michoacán. Es una zona caracterizada por condiciones de vulnerabilidad social debido a los factores de riesgo existentes como marginación, pobreza, inseguridad (desapariciones, hallazgos de cuerpos, robos, etc.) insuficiencia de servicios públicos, insuficiencia de áreas verdes y de esparcimiento para niños y adolescentes, exposición al consumo y venta de drogas legales e ilegales, violencia, entre otros. No obstante, lo que suele ser muy evidente es la exposición constante al consumo de drogas y alcohol.

\subsection{Instrumentos}

Para llevar a cabo la medición de las variables de estudio se utilizaron seis instrumentos cuantitativos.

a. Escala Tolerancia a la frustración (Bar-On, 2000), está conformada por ocho ítems, con alternativas de respuesta tipo Likert del 1 al 5 , el índice alfa de Cronbach es de $\alpha=.77$.

b. Escala de Autoestima (Rosenberg, 1965), la cual es una escala unifactorial, se compone 
de 10 ítems que evalúan contenidos sobre los sentimientos de respeto y aceptación de si mismo/a. El índice de consistencia interna es de $\alpha=.77$

c. Cuestionario Conflictack (Kimsey \& Fuller, 2003), es una escala que mide los estilos de resolución de conflictos en jóvenes y adolescentes. Está conformada por 18 ítems que se dimensionan en tres factores: Resolución cooperativa (centrado en el problema, interés por la causa del conflicto), Resolución evitativa (los conflictos deben evitarse) y Resolución agresiva (centrado en sí mismo, las cosas se hacen a la manera propia). Los coecientes alfa de Cronbach para el instrumento en su conjunto es de $\alpha=.84$; de manera particular, los índices de los factores son adecuados también, Resolución cooperativa tiene un alfa de $\alpha=.90$, Resolución agresiva de $\alpha=.77$, y la Resolución evitativa presenta una consistencia interna de $\alpha=.70$.

d. Escala de habilidades comunicativas en familia (Morales, 2014), mide los factores Apoyo y unión, Habilidades asertivas, Acuerdos, Manejo de reglas y Compromiso afectivo a través de 20 ítems con cuatro opciones respuestas, el índice de consistencia interna total de la escala es de $\alpha=.92$.

e. Escala de resistencia a la presión de amigos para el consumo de alcohol (Sánchez et al., 2013), permite evaluar la actitud o disposición hacia el alcohol, es una escala unifactorial que está compuesta por 6 ítems con opciones de respuesta tipo likert y cuyo índice de confiabilidad es de $\alpha=.78$

f. Escala de actitud ante las drogas (García del Castillo et al., 2000), fue utilizada para medir la actitud hacia las drogas, se compone de 13 ítems dimensionados en tres categorías: predisposición para uso y consumo de drogas, percepción de riesgo y percepción de satisfacción por el consumo de drogas. El índice alfa de Cronbach de los componentes oscila entre $\alpha=.67$ y $\alpha=.74$.

\subsection{Técnicas de recolección de información}

La información cuantitativa fue complementada con una entrevista semiestructurada, la cual fue aplicada al director de la institución educativa; tal técnica fue llevada a cabo en dos sesiones de trabajo; así mismo, se utilizó la observación del medio escolar y del contexto social a fin de corroborar la información vertida en la entrevista. Tales técnicas permitieron complementar la información en la fase de preevaluación, al aportar datos sobre las necesidades no solo de los adolescentes participantes, sino también de los ámbitos involucrados y con ello planear la estrategia de intervención de la mejor manera.

\subsection{Procedimiento}

El trabajo empírico inició con la determinación de la muestra de estudio, dada la necesidad que presentó el director de la institución, al sugerir que los alumnos de nuevo ingreso fueran los beneficiados para recibir la intervención, posteriormente se llevó a cabo la entrevista con él, lo que permitió contar con un panorama más amplio de las necesidades de los participantes, así como de la comunidad educativa inserta en un contexto social de vulnerabilidad. De igual forma se llevó a cabo la observación dentro de las aulas, fuera de ellas y en la comunidad, haciendo recorrido de sus calles. 
El proceso se proyectó en tres fases, no obstante para implementarlo, se procedió a enviar a los padres el consentimiento informado a fin de solicitar el permiso para que sus hijos participaran en el estudio; una vez que se contó con los formatos firmados por los padres se llevó a cabo la primera de las fases, la preevaluación, esta se logró a través de la aplicación de los instrumentos dentro de las aulas, dicho proceso se llevó a cabo en dos sesiones diferentes debido a la cantidad de medidas a aplicar. Cabe aclarar que se incluyó el constructo autoestima como eje rector en la toma de decisiones y bienestar personal, así como la comunicación familiar ya que la convivencia positiva en el hogar es fundamental para mitigar los efectos de factores de riesgo externos. Posterior a la aplicación, se hizo el llenado de los datos y se procedió a su análisis para detectar las necesidades de la población de estudio.

La segunda fase corresponde al diseño e implementación del programa de intervención con base en las necesidades detectadas no solo mediante los resultados arrojados por los instrumentos, sino también a través de la observación y la entrevista con el director. El programa fue realizado bajo la modalidad de taller, incluyéndose 12 sesiones de 90 minutos cada una, una vez por semana (ver Tabla 1).

Tabla 1. Sesiones del Programa de intervención

\begin{tabular}{|c|c|}
\hline \# sesión & Temas \\
\hline 1 & Autoconcepto y autoestima \\
\hline 2 & Tolerancia a la frustración \\
\hline 3 & Conductas riesgo: adicciones \\
\hline 4 & Autocontrol como estrategia frente a las adicciones \\
\hline 5 & Relaciones sociales positivas \\
\hline 6 & Asertividad y toma de decisiones \\
\hline 7 & Manejo de conflictos \\
\hline 8 & Conflictos: negociación y mediación \\
\hline 9 & Adicción al alcohol y drogas \\
\hline 10 & Inteligencia emocional y adicciones \\
\hline 11 & Comunicación familiar como factor protector \\
\hline 12 & ¿Qué hacer frente a la exposición de drogas y alcohol? \\
\hline
\end{tabular}

Fuente: Elaboración propia (2020). Tomado de Programa de Intervención "Prevención de conductas de riesgo en adolescentes a través de desarrollo de habilidades para la vida"

Cada sesión de trabajo se dividió en cuatro momentos; el primero, introductorio donde se sensibilizaba al grupo, se hacía una reflexión sobre el tema anterior o se preguntaba por las actividades de tarea; el segundo momento fue caracterizado por el manejo de dinámicas grupales acorde a la temática de la sesión y su respectiva reflexión; en la tercera parte se abordó el contenido teórico de cada uno de los temas, utilizando distintas técnicas didácticas, y la última parte se destinó al cierre de sesión, optando por una retroalimentación grupal y espacio para la reflexión de lo aprendido. La tercera fase constituye la postevaluación, la cual se realizó una vez concluida la intervención, al aplicar los mismos instrumentos para evaluar las variables de estudio, lo cual se llevó a cabo en las aulas de manera colectiva, en dos sesiones como en la primera fase. 


\subsection{Análisis de datos}

Una vez que se contó con la información de ambas aplicaciones, se elaboró la base de datos y posteriormente se analizaron mediante el SPSS 21.0, utilizando t de Student y coeficiente de correlación de Pearson para identificar la existencia de relación entre la resolución de conflictos y la actitud hacia las drogas y el alcohol. La t de Student permitió comparar las variables de estudio antes y después de la implementación del programa de intervención. Dichos datos fueron triangulados con la información cualitativa.

\subsection{Consideraciones éticas}

Como elemento fundamental de las consideraciones éticas, se entregó el consentimiento informado a los padres de familia que permitiera la participación de sus hijos e hijas en el programa de intervención, donde se puntualizó el carácter de la confidencialidad de los datos y el respeto por el anonimato de los participantes.

\section{Resultados}

Como respuesta al objetivo general, tendiente a desarrollar habilidades socioemocionales asociadas a la resolución de conflictos a partir de la implementación de un programa de intervención, los hallazgos muestran que posterior a su implementación, se observaron cambios estadísticamente significativos en Autoestima y Tolerancia a la frustración, destacando un mejoramiento revelador principalmente en esta última variable, como se muestra en la Figura 1.

Figura 1. Cambios en Autoestima y Tolerancia a la frustración posterior a la intervención

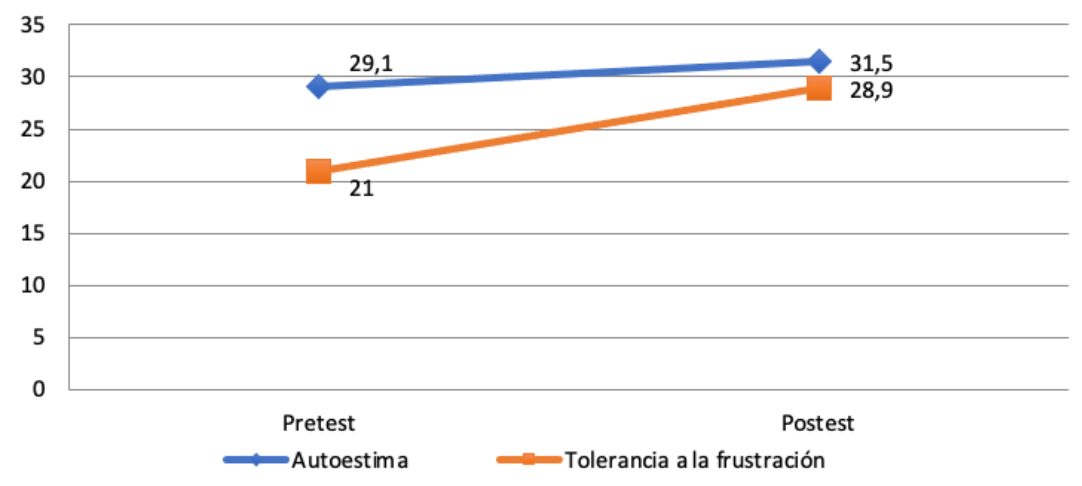

Fuente: Elaboración propia (2020).

En torno a la resolución de problemas, los cambios observados posterior a la implementación del programa se registraron en dos de los tres factores evaluados, disminuyó el uso de los estilos agresivo y evitativo como forma de solucionar un problema. No se reportan cambios en el estilo de resolución cooperativa (Ver Figura 2). 
Figura 2. Cambios en Resolución de problemas posterior a la implementación del programa

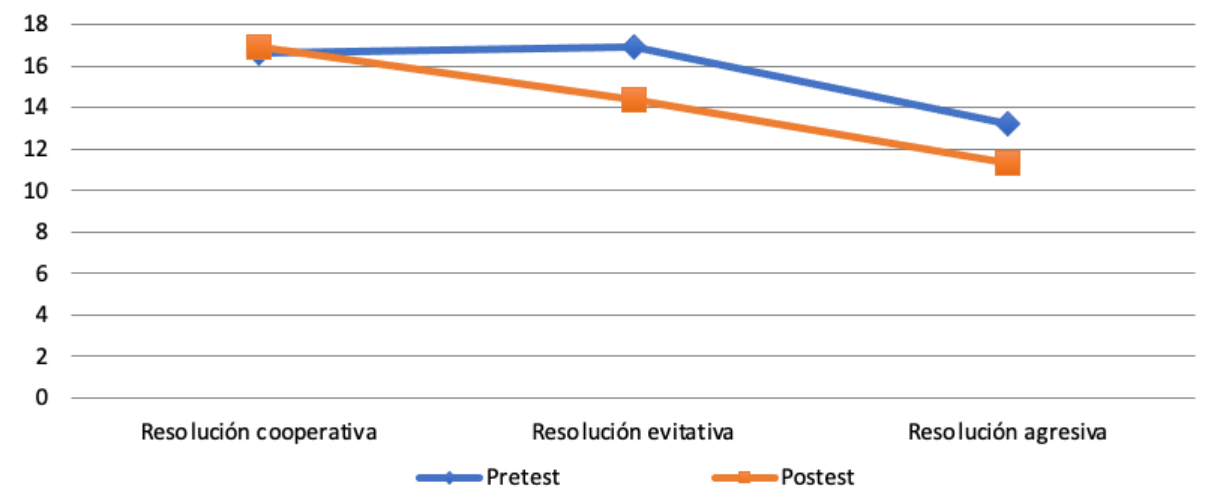

Fuente: Elaboración propia (2020).

La Actitud ante las drogas y la Resistencia a la presión para consumo de alcohol mostraron cambios estadísticamente significativos, tal y como se muestra en la Figura 3. En ambas variables hubo un cambio de actitud posterior a la implementación del programa, es decir, disminuyó la aceptación hacia el consumo de drogas y mejoró la resistencia a la presión de los iguales para el consumo de alcohol.

Figura 3. Cambios en actitud hacia las drogas y alcohol después de la intervención

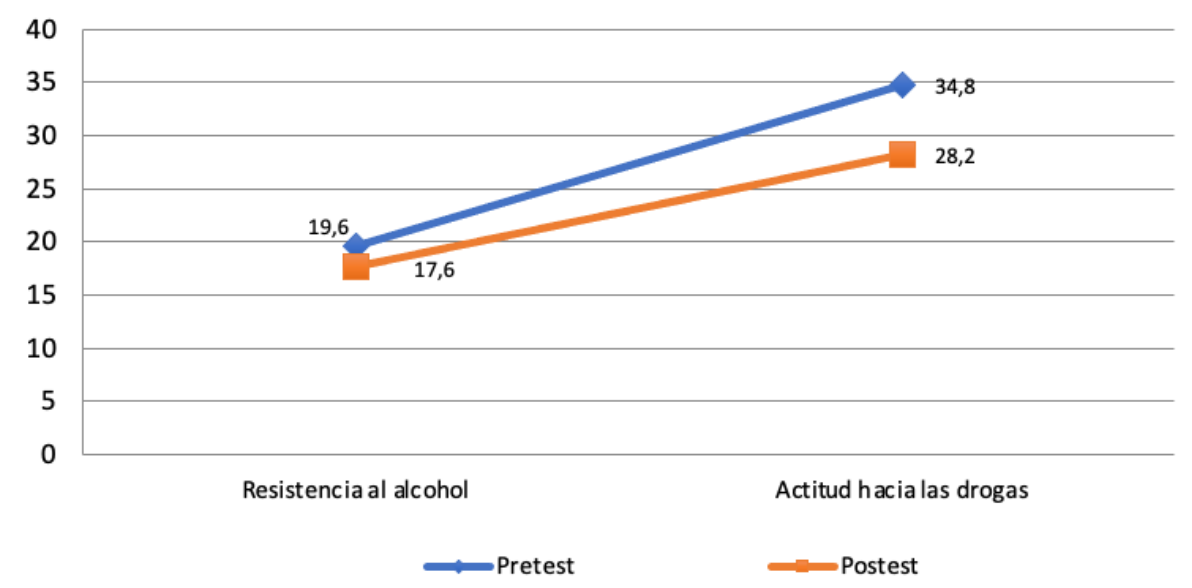

Fuente: Elaboración propia (2020).

La comunicación familiar, que permitió evaluar habilidades sociales, mostró cambios favorables solamente en el factor habilidades asertivas ( $\mathrm{t}=2.17$; $\mathrm{p}<.040)$ como resultado de la implementación del programa, lo que significa que los adolescentes refirieron que, en casa, aprendieron a expresar pensamientos y sentimientos de manera respetuosa. El resto de los factores no mostraron cambios estadísticamente significativos.

Con el propósito de dar respuesta a uno de los objetivos particulares del estudio, se llevó a cabo un análisis correlacional, lo cual arrojó la existencia de asociación entre la resolución de problemas y la actitud hacia las drogas y el alcohol, en particular la resolución cooperativa y evitativa; lo que significa que, a mayor uso de un estilo evitativo de resolución de problemas 
y menor uso de un estilo cooperativo, mayor será la aceptación hacia el consumo de alcohol y drogas (Ver Tabla 2).

Tabla 2. Correlaciones entre actitud hacia las drogas y el alcohol y resolución de conflictos

\begin{tabular}{|c|c|c|c|}
\hline & Resolución Cooperativa & Resolución Evitativa & Resolución Agresiva \\
\hline Actitud alcohol & $-.270^{* * *}$ & $.326^{* *}$ & $.127^{* *}$ \\
\hline Actitud drogas & $-.433^{* * *}$ & $.309^{* *}$ & .086 \\
\hline
\end{tabular}

Nota: **p<.01 Fuente: Elaboración propia, 2020.

Por otro lado, a fin de corroborar si las habilidades socioemocionales se asocian con la manera de resolver los conflictos, se llevó a cabo el análisis correlacional correspondiente, destacando efectivamente la relación entre las variables. En torno al componente emocional, como se muestra en la Tabla 3, a mayor autoestima y tolerancia a la frustración, mayor utilización de estrategia cooperativa de problemas, y menor uso de estrategias evitativa y agresiva.

Tabla 3. Correlaciones entre Resolución de problemas y habilidades emocionales

\begin{tabular}{lccc} 
& \multicolumn{3}{c}{ Resolución de problemas } \\
\cline { 2 - 4 } Habilidades socioemocionales & $\begin{array}{c}\text { Resolución } \\
\text { cooperativa }\end{array}$ & Resolución evitativa & Resolución agresiva \\
\hline Autoestima & $\mathbf{3 0 5 ^ { * * * }}$ & $-\mathbf{. 2 5 0 ^ { * * * }}$ & $\mathbf{- . 2 2 5 ^ { * * * }}$ \\
Tolerancia a la frustración & $\mathbf{. 2 7 5 ^ { * * }}$ & $-.170^{* * *}$ & $\mathbf{- . 4 2 9 *}$ \\
\hline
\end{tabular}

Fuente: Elaboración propia (2020)

En lo que respecta a la asociación entre habilidades sociales, evaluadas mediante la comunicación familiar y resolución de problemas, se observa que, a mayor apoyo y unión, habilidades asertivas, acuerdos y compromiso afectivo en la familia, mayor uso de resolución cooperativa de problemas y menor utilización de resolución evitativa y agresiva (Tabla 4).

Tabla 4. Correlaciones entre Resolución de problemas y habilidades sociales

\begin{tabular}{lccc} 
& \multicolumn{3}{c}{ Resolución de problemas } \\
\cline { 2 - 4 } Comunicación familiar & $\begin{array}{c}\text { Resolución } \\
\text { cooperativa }\end{array}$ & Resolución evitativa & Resolución agresiva \\
\hline Apoyo y unión & $.260^{* *}$ & $-.385^{* * *}$ & $-.230^{* *}$ \\
Habilidades asertivas & $.365^{* *}$ & $-.301^{* * *}$ & $-.390^{* *}$ \\
Acuerdos & $.356^{* *}$ & $-.301^{* * *}$ \\
Manejo de reglas & $.285^{* *}$ & $-.231^{* * *}$ \\
Compromiso afectivo & & $-.329^{* *}$ & $-.412^{* * *}$ \\
\hline
\end{tabular}

Fuente: Elaboración propia (2020) 
En relación a la información obtenida cualitativamente, un dato significativo vertido en la entrevista de diagnóstico con el director evidenció que existían algunos alumnos que habían sido sorprendidos con droga en el baño, más no consumiéndola. En una entrevista breve posterior a la intervención, afirmó que los alumnos cambiaron la actitud hacia estas, ya que, en los pasillos de la escuela, o en el aula ya no hacían comentarios al respecto e incluso se habían acercado al director 3 de los 4 alumnos sorprendidos, para informarle que reconocían el error de llevar droga a la escuela, que lo habían hecho para adquirir un rol popular entre los compañeros, pero que concebían que no era la manera idónea para obtenerlo.

A lo largo de las sesiones de trabajo pudo observarse que al inicio hacían comentarios a favor del consumo e incluso de venta de drogas, que en la comunidad era común observar personas consumiendo alcohol y drogas. Conforme avanzó la intervención, expresaron que sumirse en el mundo del alcohol y las drogas, no era una forma correcta de resolver problemas familiares, escolares y personales, sino solo era una manera de evadirlos y que, por lo tanto, el problema seguiría. Manifestaron que a pesar de que en su comunidad seguía existiendo una exposición constante al consumo de drogas y alcohol, ellos tenían la posibilidad de elegir formas más saludables de enfrentar los conflictos.

\section{Discusión}

Los comportamientos de riesgo, incluido el uso de sustancias, comúnmente inician en la adolescencia temprana (Kann et al., como se cita en Houck et al., 2016), etapa en la que se ubican los participantes del estudio. Por lo tanto, se requiere potenciar recursos personales para que afronten hábilmente los desafíos diarios. Las habilidades socioemocionales son capacidades que incluyen el manejo de sentimientos- su reflexión y autorregulación, la comunicación eficaz, empatía, confianza y autoeficacia, planificación y resolución de problemas, gestión de conflictos, creatividad, determinación y autodisciplina (Clarke et al., 2015), las cuales son recursos psicológicos fundamentales que favorecen la toma asertiva de decisiones.

En este sentido, los cambios observados en las habilidades socioemocionales evaluadas, desmuestran que la intervención promovió una mejora en la autoestima. Lo anterior favorece lo que Montoya y González-Arratia (como se cita en Silva \& Mejía, 2015), conciben como apreciación de sí mismo, mediante un proceso valorativo-acumulativo, basado en la experiencia cotidiana y enfocado a un yo actual, pero influenciado por estados afectivos. Lo anterior permite entender que los adolescentes aprendieron a valorarse en función de sus propias cualidades.

En la misma línea, hubo una mejora significativa en la tolerancia a la frustración, aumentando la capacidad para resistir a situaciones adversas. La tolerancia a la frustración es la capacidad para soportar situaciones y sucesos difíciles y estresantes, en la que la persona demora su respuesta, independientemente de la que sea, y continúa aún cuando resulte displacentero (Ventura et al., 2018); se ha reportado que la falta de tolerancia a la frustración genera problemas emocionales (Mustaca, 2015). Con base en los resultados, los adolescentes desarrollar la capacidad de lidiar con eventos estresantes y aprendieron a tolerarlos, aspecto que les permite resolver situaciones conflictivas de manera más adecuada. Los cambios observados en la forma de resolver los conflictos evidencian que los adolescentes identificaron que evadir los problemas o resolverlos 
de manera agresiva, no son estrategias que les traigan beneficios, al contrario, tienden a perpetuarlos.

Resolver de manera agresiva indica que la persona quiere que las cosas se hagan a su manera, actuando de manera agresiva y autoritaria, con una actitud egoísta donde solo le interesa satisfacer sus propias necesidades incluso a expensas de los demás. En tanto, actuar de manera evitativa se basa en la creencia de los conflictos deben evitarse debido a que generan malestar, por lo tanto, se actúa de manera pasiva ante el conflicto (Garaigordobil et al., 2016). El desarrollo de competencias para el manejo constructivo de conflictos constituye un elemento esencial en la educación de los adolescentes, que, a su vez, favorece mejores formas de convivencia (Carreño \& Rozo, 2020). Si aprenden a identificar que los estilos agresivo y evitativo siguen manteniendo los conflictos, habrán dado el primer paso, que es optar por alejarse de tales formas de resolución en la búsqueda de formas más eficaces y saludables.

El programa de intervención se enfocó a desarrollar habilidades socioemocionales y de resolución de conflictos para disminuir conductas de riesgo y en este sentido, el objetivo se logró ya que los cambios observados en la actitud hacia el alcohol y las drogas fueron significativos, principalmente hacia las drogas. En lo que respecta a la actitud hacia el alcohol, Sánchez et al., (2013) afirman que, en la etapa adolescente, el grupo de amigos se convierte en una de las áreas más importantes de desarrollo social, y el pertenecer a un grupo se convierte en una prioridad, lo cual es adaptativo; no obstante, también puede ser fuente de riesgo si el grupo presiona para que lleve a cabo conductas que pongan en riesgo su salud física y psicológica, como es el caso del consumo de alcohol.

Si el adolescente es capaz de lidiar con la presión de los iguales para el consumo de alcohol y asumir una actitud de desagrado, favorecido por una mejora en la autoestima, estará protegiéndose del riesgo que significa iniciar en esta travesía. Como ya fue señalado, el alcohol es la sustancia legal mas utilizada por población adolescente, y dada su condición, es de fácil acceso. Aprender a lidiar frente a las presiones de otros, y reconocer que no es la vía idónea para resolver problema o para disfrutar, es fundamental para un cambio de actitud. El consumo de drogas se inicia a edades cada vez más tempranas (UNODC, 2021). El abuso de sustancias conlleva numerosos riesgos en diferentes áreas de la vida del adolescente, que puede incluso terminar con su vida.

Diversos estudios han demostrado que el grupo poblacional más expuesto al riesgo de consumo de drogas es entre los 12 y 29 años; además se sabe que en esta etapa los adolescentes por su condición física, sus expectativas e inicio a la integración social, están en mayor riesgo de involucrarse en conductas de riesgo como el consumo de drogas, lo que trae como consecuencia alteraciones en las diversas áreas de desarrollo, deteriorando su calidad de vida (Guzmán et al., 2014).

Cuando el adolescente muestra una actitud favorable hacia el consumo de drogas en más probable que se involucre a una edad temprana. Los cambios observados a partir de la implementación del programa de intervención refieren una mejora significativa, lo que significa que los adolescentes desarrollaron una actitud de mayor rechazo hacia el consumo de drogas, lo cual representa un logro importante debido a las condiciones tan adversas presentes en su comunidad. A través 
de lo manifestado en las sesiones del programa, los adolescentes reconocieron que el consumo de sustancias ilícitas es una manera de evadir la realidad que viven, quizás una realidad turbia, Ilena de obstáculos y tensiones pero que es posible lidiar con los riesgos diarios. Según MoretaHerrera et al., (2017), si existen actitudes favorables hacia las drogas, ello tiende a distorsionar la percepción de riesgo; esto es, la valoración que se tiene de una sustancia y los juicios de valor hacia la acción de consumo, son factores que predisponen a la práctica del consumo, convirtiéndose en un círculo vicioso.

En lo que respecta a la relación inversa entre habilidades emocionales y actitud hacia las drogas y hacia el alcohol, deja claro que cuanto mayores habilidades socioemocionales desarrolle el adolescente, se enfrentará de manera más eficiente a disponer de drogas y consumirlas. Algunos estudios que se enfocaron en estudiar la autorregulación de las emociones y el comportamiento en la adolescencia temprana concluyeron que existen asociaciones predictivas del manejo afectivo y el consumo de sustancias (Hessler \& Katz; Raffaelli \& Crockett, como se cita en Houck et al., 2016), lo que confirma los hallazgos.

En tanto, la relación entre resolución de conflictos y actitud hacia el consumo de drogas y alcohol refleja que cuanto más eficaz es el estilo de resolver los problemas por parte del adolescente, menos acepta el consumo de drogas como una conducta adecuada y esperada.

Las correlaciones halladas entre habilidades sociales en torno a la comunicación familiar y actitud hacia las drogas y hacia el alcohol, muestran que, si se cuenta con habilidades sociales, menos se acepta el consumo de sustancias lícitas e ilícitas. El compromiso en la relación paterno-filial y el control conductual de los padres (límites y reglas) se asocia negativamente con el consumo de sustancias psicoactivas en adolescentes (Moreno \& Palomar, 2017). En cambio, la percepción de carencia de afecto y apoyo en casa representa un factor crítico que se asocial con el consumo de alcohol y drogas en hijos adolescentes (Musitu et al., 2015).

Los resultados obtenidos confirman hallazgos de estudios previos, destacando que la promoción de habilidades socioemocionales constituye una estrategia adecuada para disminuir conductas de riesgo y potenciar factores protectores en adolescentes que viven en condiciones desfavorables socialmente.

\section{Conclusión}

Con base en los hallazgos, el programa de intervención fue efectivo en la medida en que propició cambios favorables en las actitudes y comportamiento de los adolescentes. La capacidad de resolución de conflictos es una estrategia útil para afrontar las adicciones en los adolescentes. La resolución es en sí misma una habilidad para la vida, dentro de las cuales se encuentran las habilidades socioemocionales.

Las habilidades socioemocionales mitigan el riesgo ante las conductas de riesgo y en particular, las adicciones. Una manera de promoverlas es mediante programas de intervención. Los hallazgos muestran una mejora muy significativa en autoestima, tolerancia a la frustración y en la actitud hacia el alcohol y las drogas. En el caso de la comunicación familiar, la mejora se observó en habilidades asertivas. La resolución de conflictos se vio mejorada en la respuesta agresiva y evitativa, disminuyendo sus niveles. 
A la luz de los resultados, se concluye que promover habilidades socioemocionales y de resolución de conflictos genera un impacto en la actitud hacia las drogas y hacia la presión sobre el uso del alcohol, promoviendo una actitud más realista y adaptativa. Los programas de intervención en ambientes educativos son una estrategia efectiva para fomentar comportamientos saludables en la adolescencia, y con ello, actuar de manera preventiva ante la inminente presencia de factores de riesgo, como son los contextos de vulnerabilidad social. Ello provee de recursos para enfrentar la disponibilidad ante el consumo de sustancias lícitas e ilícitas antes de convertirse en un grave problema de salud pública como son las adicciones al alcohol y las drogas.

No cabe duda que la información que se reporta también refleja las limitaciones a las que se enfrentó a lo largo del trabajo de campo; una de estas es la cantidad de participantes, lo que limita la posibilidad de generalizar los datos, al ser una muestra reducida. El tipo de diseño es otra de las limitantes ya que para contar con un proceso metodológico más robusto conviene realizar diseños experimentales puros. Otra de las limitantes halladas es la imposibilidad de confirmar los cambios registrados, ya que el estudio es transversal, por lo que, para verificar la eficacia de la intervención, y como futras líneas de acción, conviene proyectar un estudio longitudinal que muestre si la actitud hacia las drogas y alcohol se mantiene, o se modifica de acuerdo con condiciones externas e incluso aquellas propias del adolescente.

\section{Referencias}

90 grados. (2019, 28 de marzo). Según el INEGI en Morelia, Michoacán 70 por ciento de la Población se siente insegura. 90 grados. Agencia Mexicana de Noticias. https://cutt.ly/BYzd4IQ

Alarcón, C., Arévalo, M., Torres, I., Soto, L., \& Cardona, J. (2015). Motivaciones y recursos para el consumo de sustancias psicoactivas en universitarios. Hacia la Promoción de la Salud, 17(1), 92-104. https:// revistasojs.ucaldas.edu.co/index.php/hacialapromociondelasalud/article/view/2025

Aranda, M. G., \& Caldera, J. F. (2018). Gamificar el aula como estrategia para fomentar habilidades socioemocionales. Revista educarnos, 8(31), 41-66. https://revistaeducarnos.com/wp-content/ uploads/2018/11/educarnos31-1.pdf \#page $=41$

Bar-On, R. (2000). The Bar-On Emotional Quotient Inventory: Youth Version (EQ-I: YV) Technical Manual. MultiHealth Sys-tems, Inc.

Berdullas, S. S. (2016). Relación entre las habilidades de solución de problemas sociales y los rasgos emocionales negativos de la personalidad: la "triada emocional negativa" (depresión, ansiedad e ira). [Disertación doctoral]. Universidad Complutense de Madrid. https://eprints.ucm.es/id/eprint/37692/

Bisquerra, R. (2003). Educación emocional y competencias básicas para la vida. Revista de investigación educativa, 21(1), 7-43. https://revistas.um.es/rie/article/view/99071

Carreño, M. \& Rozo, H. (2020). Estrategias para desarrollar la convivencia y la paz desde la educación. Academia y Virtualidad 13(2), 35-56. http://dx.doi.org/10.18359/ravi.4501

Clarke, A.M., Morreale, S., Field, C., Hussein, Y. y Barry, M. (2015). What works in enhancing social and emotional skills development during childhood and adolescence? A review of the evidenceon the effectiveness of schoolbased and out-of-school programmes in the UK. World Health Organization Collaborating Centre for Health Promotion Research, National University of Ireland Galway, National University of Ireland Galway. https://cutt.ly/yYzfNFA

Duckworth, A., y Yeager, D. (2015). Measurement matters: Assessing personal qualities other than cognitive ability for educational purposes. Educational Researcher, 44(4), 237-251. https://doi. org/10.3102\%2Fo013189X15584327 
Garaigordobil, M. (2008). Intervención psicológica con adolescentes: un programa para el desarrollo de la personalidad y la educación en derechos humanos. Pirámide.

Garaigordobil, M., Machimbarrena, J., y Maganto, C. (2016). Adaptación española de un instrumento para evaluar la resolución de conflictos (Conflictalk): Datos psicométricos de fiabilidad y validez. Revista de Psicología Clínica con Niños y Adolescentes, 3(2), 59-67. https://www.revistapcna.com/sites/default/ files/16-23.pdf

García, B. (2018). Las habilidades socioemocionales, no cognitivas o "blandas": aproximaciones a su evaluación. Revista Digital Universitaria (RDU), 19(6). http://doi.org/10.22201/codeic.16076079e.2018. v19n6.a5

Guzmán, F., García, B., Rodríguez, L. \& Alonso, M. (2014). Actitud, norma subjetiva y control conductual como predictores del consumo de drogas en jóvenes de zona marginal del norte de México. Frontera Norte, 26(51), 53-74. https://doi.org/10.17428/rfn.v26i51.556

Hernández, O., Espada, J. y Guillén, A. (2016). Relación entre conducta prosocial, resolución de problemas y consumo de drogas en adolescentes. Anales de Psicología, 32(2), 609-616. https://doi.org/10.6018/ analesps.32.2.204941

Hernández-Samperi, R. Fernadez-Collado, C., Baptista-Lucio, M. (2010). Metodología de la investigación. Mac Graw Hill.

Houck, C., Hadley, W., Barker, D., Brown, L., Hancock, E., \& Almy, B. (2016). An Emotion Regulation Intervention to Reduce Risk Behaviors Among at-Risk Early Adolescents. Prevention Science, 17(1), 71-82. https://doi.org/10.1007/s11121-015-0597-0

Kimsey, W. D., \& Fuller, R. M. (2003). Conflictalk: An instrument for measuring youth and adolescent conflict management message styles. Conflict Resolution Quarterly, 21(1), 69-78. https://doi.org/10.1002/crq.49

García del Castillo, J., Dias, P., Díaz-Pérez, J., Bastos, A. S., del Castillo-López, A., López-Sánchez, C., \& Maciá, D. (2000). Adaptación de las escalas de actitudes hacia el tabaco, el alcohol y otras drogas en adolescentes. Salud y Drogas, 12(1), 79-99. https://doi.org/10.21134/haaj.v12i1.11

Marrero, O., Mohamed, R., y Xifra, J. (2018). Habilidades blandas: necesarias para la formación integral del estudiante universitario. Revista Científica ECOCIENCIA, 5, 1-18. https://doi.org/10.21855/ ecociencia.50.144

Melo, D., y Castanheira, L. (2010). Autoeficacia y actitud hacia el consumo de drogas en la infancia: explorando los conceptos. Rev. Latino-Am. Enfermagem, 18, 655-62. https://doi.org/10.1590/So10411692010000700024

Merino, C. (2012). Forma breve del SPSI_R: Análisis preliminar de validez interna y confiabilidad. Terapia Psicológica, 30(2), 85-90. http://teps.cl/index.php/teps/article/view/127

Mónaco, E., Barrera, U., \& Montoya, I. (2017). Desarrollo de un programa de intervención para mejorar las competencias emocionales, el afecto positivo y la empatía en la adolescencia. Calidad de vida y Salud, 10(1), 41-56. http://revistacdvs.uflo.edu.ar/index.php/CdVUFLO/article/view/146

Morales, M. (2014). Escala de habilidades comunicativas en familia desde la perspectiva del adolescente: resultados preliminares. Memoria en extenso del XLI Congreso Nacional del CNEIP. Mazatlán, México.

Moreno y Palomar, J. (2017). Factores familiares y psicosociales asociados al consumo de drogas en adolescentes. Interamerican Journal of Psychology, 51(2), 141-151. https://doi.org/10.30849/rip/ijp.v51i2.31

Moreta-Herrera, R., Mayorga-Lascano, M., León-Tamayo, L. \& Ilaja-Verdesoto, B. (2017). Consumo de sustancias legales, ilegales y fármacos en adolescentes y factores de riesgo asociados a la exposición reciente. Salud y drogas, 18(1), 39-50. https://doi.org/10.21134/haaj.v18i1.333 
Moreta, R., Reyes, C., Mayorga, M., y León, L. (2017). Estimación sobre niveles y factores de riesgo psicosocial en adolescentes escolarizados de Ambato, Ecuador. Pensando Psicología, 3(22), 29-40. https://doi.org/10.16925/pe.v13i22.1986

Mustaca, A. E. (2015). Terapias con apoyo empírico. Relaciones con la ciencia básica. PSIENCIA. Revista Latinoamericana de Ciencia Psicológica, 7(1), 80-87.

Musitu, G., Suárez, C., Del Moral, G., \& Villarreal, M. (2015). El consumo de alcohol en adolescentes: el rol de la comunicación, le funcionamiento familiar, la autoestima, el consumo en la familia y amigos. Revista Búsqueda, 14, 45-61. https://doi.org/10.21892/01239813.58

Ortiz-Ruiz, N. y Díaz-Grajales, C. (2018). Una mirada a la vulnerabilidad social desde las familias. Revista Mexicana de Sociología, 80(3), 611-638. https://doi.org/10.22201/iis.01882503p.2018.3.57739

Rodríguez, C., Celma, L., Orejudo, S. \& Rodríguez, L. (2012). Desarrollo de las habilidades sociales y emocionales de los adolescentes en el aula: Programa de educación socio-emocional SEA. Tea Ediciones.

Rojas-Solís, J., Morales-Quintero, L., Juarros-Basterretxea, J., Herrero, J. y Rodríguez-Díaz, F. (2019). Propiedades psicométricas del Inventario de Estilos de Resolución de Conflictos en jóvenes mexicanos. Revista Iberoamericana de Psicología y Salud, 10(1), 15-26. https://doi.org/10.23923/j. rips.2019.01.022

Rosabal García, E., Romero Muñoz, N., Gaquín Ramírez, K., \& Hernández Mérida, R. A. (2015). Conductas de riesgo en los adolescentes. Revista Cubana de Medicina Militar, 44(2), 218-229. https://cutt.ly/oYzhtx9

Rosenberg, M. (1965). Society and the adolescent self-image. Princeton University Press.

Sánchez-González, D., \& Egea-Jiménez, C. (2011). Enfoque de vulnerabilidad social para investigar las desventajas socioambientales. Su aplicación en el estudio de los adultos mayores. Papeles de Población, 17(69), 151-185. https://rppoblacion.uaemex.mx/article/view/8463

Sánchez, C., Andrade, P., Betancourt, D. \& Vital, G. (2013). Escala de Resistencia a la Presión de los Amigos para el Consumo de Alcohol. Acta de Investigación Psicológica, 3(1), 917-929. http://doi.org/10.1016/ S2007-4719(13)70942-6

Silva, I. y Mejía, O. (2015). Autoestima, adolescencia y pedagogía. Revista Electrónica Educare, 19(1), 24-256. https://doi.org/10.15359/ree.19-1.13

Treviño, D., González, M., y Montemayor, K. (2019). Habilidades socioemocionales y su relación con el logro educativo en alumnos de Educación Media Superior. Revista de Psicología y Ciencias del comportamiento de la Unidad Académica de Ciencias Jurídicas y Sociales, 10(1), 32-48. https://doi. org/10.29059/rpcc.20190602-79

UNODC (2021). World Drug Report 2020. United Nations publication. https://cutt.ly/aYzhP9Y

Valdés, A., y Ochoa, J. (2010). Familia y crisis. Pearson.

Ventura, J., Caycho, T., Vargas, D., y Flores, G. (2018). Adaptación y validación de la Escala de Tolerancia a la Frustración (ETF) en niños peruanos. Revista de Psicología Clínica con Niños y Adolescentes, 5(2), 23-29. http://doi.org/10.21134/rpcna.2018.05.2.3

West, R. (2016, marzo 17). Should non-cognitive skills be included in school accountability systems? Preliminary evidence from California's core Districts. Bookings. https://cutt.ly/TYzhG87

\section{AUTORA}

Marisol Morales Rodríguez. Doctora en Ciencias de la educación, Máster en Psicología de la Salud. Profesora Investigadora de tiempo completo. Coordinación de Planeación institucional, Facultad de Psicología, UMSNH. 